\title{
Validade e Fidedignidade da Escala com Figuras de Competência Percebida e Aceitação Social para Crianças Brasileiras
}

http://dx.doi.org/10.11606/1807-5509202000020331
Nadia Cristina VALENTINI* Paulo Felipe Ribeiro BANDEIRA* Mary Elizabeth RUDISILL**
*Universidade Federal do Rio Grande do Sul, Porto Alegre, RS, Brazil.

**Auburn University, Auburn, Alabama, USA.

\section{Resumo}

A Pictorial Scale of Perceived Competence and Social Acceptance (PSPCSA) é uma escala utilizada para identificar as auto-percepções de competência (cognitiva e física) e aceitação social (pares e maternas) de crianças na educação infantil e primeiros anos do ensino fundamental I. 0 objetivo deste estudo foi traduzir e investigar a validade de conteúdo, critério, constructo e a fidedignidade da PSPCSA para crianças brasileiras. A tradução transcultural foi utilizada, e o processo de validação foi conduzido envolvendo 33 profissionais e 667 crianças (4 a 7 anos); 159 dessas crianças responderam a PSPCSA em dois momentos. Com base nos resultados pode-se inferir que houve clareza de linguagem e pertinência das questões, elevada consistência interna (valores > 96) e fidedignidade teste-reteste (valores de $r>$ 0,60 ). A PSPCSA apresentou ajustada adesão das questões com as correspondentes dimensões e subescalas e índices satisfatórios de validade fatorial confirmatória (Non-normed Fit Index, Comparative Fit Index e Tucker and Lewis's Index of Fit com valores $>0,81$ ). As versões brasileiras da PSPCSA apresentam indicadores de validade para o uso com crianças brasileiras.

Palavras-Chave: PSPCSA; Escala de Figuras de Auto-Percepção; Estudo de Validação.

\section{Introdução}

Perceber-se competente e aceito socialmente emerge de forma gradual na infância e de maneira distinta em diferentes domínios ${ }^{1-3}$; conforme a criança se torna consciente de suas capacidades e adquire novas habilidades ${ }^{4}$. Quando compelida a agir de forma efetiva no contexto, se a criança obtém sucesso nas tentativas, vivencia sentimentos de eficácia, os quais aumentam a motivação para a competência individual. As experiências passadas, o sucesso ou fracasso em tarefas desafiadoras, as interaçóes com outros, e o apoio de pessoas significativas nas experiências de conquista, influenciam nas avaliaçóes sobre a própria competência ${ }^{1,3}$.

A criança expressa as suas percepções de competências e de aceitação em forma de julgamentos relevantes que faz sobre suas habilidades cognitivas, físicas e sociais ${ }^{2,3,5}$. As percepções individuais coexistem em uma relação circular com a motivação; e, são mediadores importantes das conquistas ao longo da infância ${ }^{3,6,7}$. Crianças que se percebem em controle sobre o contexto, demonstram sentimentos de eficácia e de prazer intrínseco para aprender, os quais fortalecem a competência percebida ${ }^{1,2}$. Crianças que se percebem competentes evidenciam habilidades verbais assertivas ${ }^{8}$ e recursos para lidar com desafios e para preservar as percepções próprias de habilidade 6 .

Contrariamente, crianças que percebem que o controle de suas experiências é externo demonstram ansiedade nas experiências de conquistas e declínios na motivação intrínseca; os quais ao longo do tempo comprometem as percepções próprias de competência ${ }^{1}$. Crianças com a competência percebida fragilizada demonstram maior incidência de solidão, isolamento social ${ }^{9,10}$; e, de desordens 
relacionadas à ansiedade ${ }^{11}$. A exclusão por parte dos pares também tem sido reportada ${ }^{9,12}$. Destaca-se que crianças com baixas percepções de competência podem demonstrar dificuldades para entender suas emoções ${ }^{13}$ e para se adaptarem no ambiente escolar ${ }^{9}$. Além disso, atrasos em tarefas escolares ${ }^{14-17}$ e motoras ${ }^{11,18,19}$, bem como menor engajamento ${ }^{12}$ e motivação para aprender ${ }^{20}$ tem sido reportados nessas crianças. $\mathrm{O}$ efeito acumulativo de vários anos de baixas percepções de competência pode ter consequências significantes ao longo da vida.

Perceber-se competente é essencial para que crianças fortaleçam o engajamento em tarefas escolares $^{12}$ e a motivação intrínseca para aprender ${ }^{19,20}$; demonstrem esforços frente as dificuldades e novos desafios ${ }^{19}$; resolvam problemas de maneira independente no ambiente escolar ${ }^{20}$; desenvolvam relações efetivas com pares ${ }^{12}$; fortaleçam a autoestima $^{16}$ as conquistas motores ${ }^{19}$ e as relações sociais $^{12,16}$. Portanto, investigar este fenômeno, ao longo da infância, é essencial para a compressão dos padrões de motivação adotado pela criança em diferentes ambientes de conquista (que envolvem a competição com pares e/ou a auto superação) e de aprendizagem. A abordagem multidimensional do estudo da competência percebida permite também identificar em qual domínio (cognitivo, social ou motor) a criança está investindo maior energia e esforço para tornar-se competente.

$\mathrm{O}$ entendimento deste constructo permite desvelar o interesse da criança por determinada área, como agentes socializadores respondem aos esforços das crianças em momentos de conquistas, e a história pregressa de socialização da criança $a^{1-3,18,19}$. Ainda mais o entendimento de como a criança se percebe permite a intervenção profissional na implementação de ambientes que fortaleçam essas percepçôes ${ }^{3,6,18,19}$, no estabelecimentos de metas e estratégias familiares e/ou educacionais que fortaleçam o engajamento na aprendizagem, a autoconfiança e o autoconceito da criança. Os esforços profissionais podem ser dirigidos para o fortalecimento das competências individuais de crianças em diferentes domínios, com o suporte de pares e pessoas adultas significativas na vida da criança. Com o fortalecimento de competências individuais as percepçôes de crianças tendem a elevar-se alicerçada na competência real.

Entretanto, mensurar a auto-percepção em crianças é, sob o ponto de vista da aplicabilidade, extremamente complexo ${ }^{21}$. Crianças em fase de alfabetização não sabem ler ou leem de forma precária; demonstram habilidades linguísticas limitadas e períodos curtos de atenção no conteúdo; são inconsistentes nas respostas; e, tendem a responder o que é socialmente aceito ${ }^{22}$. Ainda mais, expressar julgamentos sobre si mesmo requer que a criança tenha consciência de sua própria competência e seja capaz de avaliar suas conquistas em diferentes domínios ${ }^{4}$. Estas particularidades estabelecem o desafio de usar instrumentos avaliativos que em seu delineamento tenham considerado essas características desenvolvimentistas. Uma opção reconhecida na literatura é a Pictorial Scale of Perceived Competence and Social Acceptance PSPCSA $^{5}$. A PSPCSA foi desenvolvida para avaliar a auto-percepção de crianças que ainda não possuem fluência na linguagem escrita. Esta escala é fundamentada no pressuposto de que a criança é capaz de mensurar sua competência quando guiada na observação pictórica de comportamentos relevantes e concretos. $\mathrm{O}$ uso de figuras é essencial para guiar a criança a expressar respostas significativas, pois facilita a aplicação e compreensão; requer o mínimo de habilidades linguísticas; desperta atenção e engajamento ${ }^{5,7}$.

As capacidades psicométricas da PSPCSA são reconhecidas como adequadas para crianças entre quatro e sete anos ${ }^{5,23-26}$; entretanto, a validade de construto e fidedignidade da PSPCSA é bastante limitada. Na própria validação original ${ }^{5,7}$, embora a PSPCSA possua quatro sub-escalas, somente um modelo de dois fatores foi testado e, ainda assim, o suporte para o uso independente das sub-escalas foi teoricamente proposto. Posteriormente, a validade de constructo da PSPCSA com crianças americanas foi reavaliada com aceitável resultado para o modelo de competência e aceitação social em crianças na educação infantil ${ }^{25}$ e nos anos iniciais ${ }^{27}$.

Estudos de validação em diferente culturas são ainda mais limitados. Por exemplo, a PSPCSA foi investigada em crianças Libanesas, evidenciando níveis adequados de consistência interna, entretanto, não se observou a formação dos fatores de competência e aceitação social apresentado no estudo original ${ }^{28}$. Uma versão adaptada da PSPCSA foi também validada para crianças holandesas com paralisia cerebral ${ }^{29}$. Posteriormente, essa mesma versão apresentou índices aceitáveis de consistência interna para crianças portuguesas com síndrome de Down ${ }^{21}$. Destaca-se que a maioria dos estudos conduzidos foram delineados com amostras pequenas, o que compromete a consistência interna e limita a investigação da validade de constructo. 
Até o momento no Brasil, duas escalas de auto-percepção foram validadas, o Self Perception Profile for Children ${ }^{30}$ e o Adolescent Self-Perception Profile $^{31}$. Essas escalas foram desenvolvidas para crianças e adolescentes com domínio da linguagem escrita; e, possuem dimensões mais complexas considerando as características de desenvolvimento destas fases especifica da vida. Por exemplo, o Self Perception Profile for Children ${ }^{30}$, para crianças alfabetizadas (idades de referência entre 8 e 12 anos) contém cinco sub-escalas em domínios específicos (competência escolar, aceitação social, competência atlética, aparência física e conduta comportamental) e uma sub-escala do autoconceito global. Ainda mais multidimensional, o Adolescent Self-Perception Profile 31 além dos domínios contidos na escala para crianças alfabetizadas incorpora três outros domínios, referentes as auto-percepções dos adolescentes em relação ao trabalho, a amizade e ao namoro ${ }^{31}$. Essas escalas apresentaram índices de validade e fidedignidade apropriados para o uso com crianças e adolescentes brasileiros e tem sido amplamente utilizadas em pesquisas ${ }^{30,31}$.

Entretanto, evidências das capacidades psicométricas da PSPCSA, em suas duas versões para crianças não alfabetizadas e/ou em fase de alfabetização (crianças na educação infantil e nos primeiros anos de ensino fundamental I), ainda não foram obtidas no Brasil. Relativamente pouca pesquisa tem sido realizada nacionalmente investigando as auto-percepçôes de crianças em fase pré-escolar ${ }^{18,32}$; decorrente, provavelmente, da dificuldade em implementar estudos uma vez que não dispomos de instrumentos validados. A validade de conteúdo e constructo é primordial para a clínica e a pesquisa, o uso de instrumentos não validados compromete a eficácia avaliativa do mesmo para outras culturas, que não a de sua origem $^{3,33,34}$.

No presente estudo endereçamos esta investigação para estimar indicadores de validação e ampliar a verificação dos modelo de dois e quatro fatores, propostos originalmente por HARTER e PIKE ${ }^{5,7}$. Portanto, os objetivos deste estudo foram traduzir a PSPCSA para a língua portuguesa; e, verificar (1) a validade de critério dos itens do PSPCPA quanto à clareza e pertinência, (2) consistência interna, (3) fidedignidade testereteste, e (4) a adequabilidade do modelo estrutural da PSPCSA para crianças brasileiras.

\section{Método}

\section{Amostra}

Compuseram a equipe de experts e público-alvo deste estudo 33 profissionais, sendo: (1) quatro tradutores juramentados; (2) três professores universitários, doutores com formação em psicologia; (3) sete mestres, três com formação em ciências do movimento humano e quatro em psicologia; (4) dois doutorandos e dois mestrandos, dois com formação em ciências do movimento humano e dois em psicologia; (5) nove professores, quatro professores de educação física e cinco pedagogos; e, (6) seis psicólogos. Também participaram do estudo 667 crianças provenientes de três estados, sete cidades e 25 escolas do Brasil. Inicialmente, um grupo de 20 crianças fez parte da avaliação da clareza do instrumento para o público-alvo infantil. Um segundo grupo, composto por 647 crianças, foi avaliado em sus escolas de origem. Destas 647 crianças, um terceiro grupo de 159 crianças respondeu a escala duas vezes com um intervalo de 7 a 10 dias, (reteste).
O número de participantes especialistas (tradutores, experts e público-alvo) e da amostra infantil foi fundamentada nas recomendações da literatura psicométrica que envolvem processos de validação cross-cultural $^{34-36}$; e, nos parâmetros estimativos de estabilidade para modelos fatoriais ${ }^{37}$. Quanto à etnia, autodeclarada pelos pais e/ou responsáveis legais no questionário sócio-demográfico, 256 $(54,8 \%)$ crianças foram reportadas como brancas; $131(28,05 \%)$ como pardas; 71 (15,22\%) como negras; e 9 (1,93\%) como orientais. Participaram do estudo crianças cujos pais ou representantes legais assinaram o Termo de Consentimento Livre e Esclarecido; e, que manifestaram verbalmente o desejo de participar. Todos os profissionais assinaram o termo de consentimento. A pesquisa foi aprovada pelo Comitê de Ética da universidade de origem.

\section{Instrumentos}

No presente estudo foi utilizado as duas versões da Pictorial Scale of Perceived Competence and Social 
Acceptance (PSPCSA), para crianças na educação infantil ( 4 e 5 anos) e para crianças nos primeiros anos do ensino fundamental I $(6 \text { e } 7 \text { anos })^{5,38}$. A PSPCSA avalia a competência percebida de crianças que ainda não dominam a linguagem escrita, sendo indicada para crianças entre aproximadamente $4 \mathrm{e} 7$ $\operatorname{anos}^{5,38}$. A PSPCSA é composta de dois domínios: competência e aceitação. As duas sub-escalas do domínio competência são referentes a competência percebida de crianças em tarefas cognitivas e físicas. As duas sub-escalas de aceitação da criança se referem as percepções de aceitação materna e dos pares. Cada sub-escala é composta de seis itens, constituindo um total de 24 questôes de respostas tipo Likert, com amplitude de escores para cada item entre 1 (baixa competência) e 4 (alta competência). A escala não requer que a criança leia ou realize respostas detalhadas; ao contrário, o avaliador conduz a crianças a responderem o instrumento por uma série de figuras, as quais retratam crianças realizando tarefas cotidianas. $\mathrm{O}$ avaliador solicita que a criança simplesmente discrimine entre pares de figuras com qual ela se parece mais simplesmente apontando para uma opção. As duas figuras, colocadas uma ao lado da outra, representam uma criança que é competente e a outra representa uma criança que não é tão competente. Após escolher a figura que melhor lhe represente, a criança deve decidir o quanto (muito ou pouco) ela é parecida com a criança da figura.

QUADRO 1 - PSPCSA número das questões para cada Subescala e Conteúdo.

\begin{tabular}{|c|c|c|}
\hline \multirow{2}{*}{$\begin{array}{l}\text { Dimensões } \\
\text { \& Subescalas } \\
\text { \& Itens }\end{array}$} & \multicolumn{2}{|c|}{ Conteúdo da Escala nos níveis de escolaridade } \\
\hline & $\begin{array}{l}\text { Educação Infantil } \\
\quad(4 \text { e } 5 \text { anos })\end{array}$ & $\begin{array}{c}1^{\circ} \text { e } 2^{\circ} \text { anos do Ensino Fundamental I (6 e } 7 \\
\text { anos) }\end{array}$ \\
\hline $\begin{array}{c}\text { Competência Cognitiva } \\
1,5,9,13,17,21\end{array}$ & $\begin{array}{c}\text { Competência em tarefas montar, reconhecer } \\
\text { cores, contar, identificar alfabeto e letra do } \\
\text { nome. }\end{array}$ & $\begin{array}{c}\text { Competência em tarefas escolares, números, } \\
\text { leitura, palavras, soletrar e somar. }\end{array}$ \\
\hline $\begin{array}{c}\text { Competência Física } \\
3,7,11,15,19,23\end{array}$ & $\begin{array}{c}\text { Competência em tarefas de nadar, escalar, } \\
\text { correr, saltitar e atar. }\end{array}$ & $\begin{array}{c}\text { Competência em tarefas de nadar, escalar, } \\
\text { quicar bola, saltitar e correr. }\end{array}$ \\
\hline $\begin{array}{c}\text { Aceitação Pares } \\
2,6,10,14,18,22\end{array}$ & $\begin{array}{l}\text { Percepção do grau de relacionamento com } \\
\text { pares nas brincadeiras na casa e no parque }\end{array}$ & $\begin{array}{c}\text { Percepção do grau de relacionamento com } \\
\text { pares nas brincadeiras e na iniciativa de rela- } \\
\text { cionar com pares. }\end{array}$ \\
\hline $\begin{array}{c}\text { Aceitação Materna } \\
4,8,12,16,20,24\end{array}$ & $\begin{array}{c}\text { Percepção sobre os cuidados da mãe para con- } \\
\text { sigo em tarefas de cozinhar, levar passear, ler, } \\
\text { brincar e conversar. }\end{array}$ & $\begin{array}{c}\text { Percepção sobre os cuidados da mãe para } \\
\text { consigo em tarefas cozinhar, levar passear, ler } \\
\text { conversar, brincar e permitir interação com } \\
\text { amigos. }\end{array}$ \\
\hline
\end{tabular}

Um questionário sócio-demográfico contendo informações sobre idade, sexo, etnia, nível socioeconômico foi respondido pelos pais. Uma escala likert ( 1 a 5 pontos) para clareza e pertinência de todos os itens da PSPCSA foi respondida pelos experts. Os experts e o público-alvo adulto julgaram cada item da escala quanto a pertinência teórica (considerando o item em relação ao desenvolvimento criança), e quanto a clareza na linguagem (considerando se os itens seriam entendidos com facilidade pela criança). A consistência no julgamento das opiniōes dos experts foi estabelecida considerando os escores na amplitude de um (pouquíssima clareza/pertinência) até cinco (muitíssima pertinência/clareza).

\section{Procedimentos}

A tradução do instrumento foi realizada de forma reversa e independente (tradução duplo- reversa). Inicialmente dois tradutores, tendo como língua materna o português, realizaram de forma independente a tradução da versão em inglês da PSPCSA para o português. Posteriormente, dois outros tradutores, utilizando as versóes produzidas para o português, reverteram a tradução para o inglês. Em comitê, os quatro tradutores e o principal pesquisador do presente estudo verificaram as semelhanças e discordâncias nas traduções. As modificações foram restritas e referentes a alguns termos mais usados no cotidiano da criança. Após consenso do comitê, uma versão unificada em português foi obtida.

O grupo de experts (doutores) e o grupo do público-alvo adulto (26 profissionais) testaram a validade de conteúdo da PSPCSA. A versão final unificada em português da PSPCSA foi enviada para estes profissionais, juntamente com a escala Likert. Experts e público-alvo adulto, usando a escala Likert de 5 pontos, avaliaram individualmente os itens da 
escala quanto a claridade e pertinência; e enviaram esta avaliação, preenchida com os escores para cada item da escala, ao principal pesquisador. A validade de conteúdo, utilizando-se de índices de confiabilidade, foi realizada nos escores fornecidos pelos experts e de face nos escores do público-alvo adulto.

Para a avaliação da clareza pelo público-alvo infantil, a versão em português foi aplicada em 20 crianças entre 4 e 7 anos ( 5 crianças para cada idade sendo 10 meninos e 10 meninas) com o objetivo de verificar se os itens da escala eram compreendidas pelas crianças sem ambiguidades; e, se os termos eram claros com linguagem acessível para as crianças. A avaliação ocorreu individualmente, nas escolas de origem de cada criança, o pesquisador explicou como a avaliação seria realizada; e, após a leitura de um item de exemplo e da certificação do entendimento da criança, as demais questões foram realizadas em voz alta pelo pesquisador. As crianças foram questionadas individualmente quanto à compreensão de cada questão.

No estudo original de validação HARTER e PIKE reportam os dados obtidos nos estudos com crianças da Preschool ( $\mathrm{M}=4,4$ anos), Kindergarten $(\mathrm{M}=5,5$ anos), First Grade (M=6,3 anos), e Second Grade $(\mathrm{M}=7,4$ anos). No presente estudo os dados foram coletados, compatíveis ao estudo de validação original da escala. Portanto, crianças provenientes de escolas de educação infantil em dois grupos de idade distintas (Grupo 1: $\mathrm{M}=4,5$ anos e Grupo 2: $\mathrm{M}=5,5$ anos); e, de escolas de ensino fundamental, $1^{\circ}$ ano $\left(\mathrm{M}=6,6\right.$ anos) e $2^{\circ}$ ano $(\mathrm{M}=7,5$ anos) do ensino fundamental, participaram do presente estudo. A pesquisa foi conduzida em 25 escolas de três estados brasileiros, resultando em uma amostra de 647 crianças entre 4 e 7 anos ( $M=6,02$ e DP=1,07).

Inicialmente, os diretores de escolas foram contatados pelo pesquisador que explicou os objetivos e procedimentos metodológicos da pesquisa. Para as escolas que aceitaram participar do estudo, o termo de consentimento livre e esclarecido foi enviado para a instituição e para os pais. Para cada criança, individualmente, o avaliador fornecia as instruçôes de como responder a PSPCSA. O item de exemplo era lido em voz alta, depois da resposta da criança e de certificar-se que a criança entendeu os procedimentos as demais questóes eram lidas em voz alta, e, uma a uma, respondias pela criança. Cada criança respondeu as questôes apontando para a resposta que mais as representavam, e muitas vezes a escolha destas respostas eram acompanhadas de expressões verbais que retratavam a identificação com as figuras contidas na escala (exemplos: "eu sou bem assim"; "parece comigo não sou boa com quebra cabeças"; "eu salto igual a ela"). Cada avaliação levou entre 20 e 30 minutos para cada criança. O reteste foi realizada para um grupo de 159 crianças em um intervalo de 7 a 10 dias do teste inicial.

\section{Análise dos dados}

Para a avaliação da clareza e pertinência foi utilizado o Coeficiente de Validade de Conteúdo - $\mathrm{CVC}^{39}$, com ponto de corte conservativo de $0,80^{40,41} ;$ e, o Coeficiente de Concordância de Kappa $^{42}$. Para a validade de face o percentual de concordância foi empregado. A consistência interna foi estimada usando o alpha de $\mathrm{Cronbach}^{34}$, para as sub-escalas e PSPCSA. A fidedignidade testereteste foi mensurada utilizando-se correlaçóes de Pearson e correlaçôes intra-classe, nos escores de 159 crianças avaliadas no teste e reteste ${ }^{40}$. Análises fatoriais confirmatórias com estimação de Maximum Likelihood foram conduzidas nos escores ( $\mathrm{n}=$ 647 crianças) verificando-se a adequabilidade dos modelos ${ }^{43}$, com estimativas de critérios múltiplos (Non-Normed Fit Index - NFI; Comparative Fit Index - CFI; Tucker e Lewis's Index of Fit - TLI) uma vez que cada índice apresenta diferentes forças de adequação do modelo ${ }^{41,42}$.

\section{Resultados}

\section{Dupla Tradução Reversa e Independente}

A tradução dupla, reversa e independente do PSPCSA resultou em duas versóes semelhantes as quais foram unificadas em comitê. Nas poucas palavras que se diferenciaram entre os tradutores, mante-se a palavra mais correntemente usada. $\mathrm{O}$ foco do comitê foi a adequação dos itens para a compressão pelo público-alvo infantil (4 a 7 anos).

\section{Validação de Conteúdo por Experts}

Nos scores fornecidos pelos experts, para 
cada item da PSPCSA, foram calculados os coeficientes de validade de conteúdo (CVC) para clareza e pertinência. A concordância dos experts em relação a clareza da linguagem da PSPCSA foi elevada, com amplitude de escores entre 97,9 e 99,2. Referente a pertinência, os valores foram ainda mais elevados com amplitude entre 98,2 e 100. Os Coeficientes de Concordância de Kappa foram elevados e significativos $(p<0,001)$. Os resultados estáo apresentados na TABELA 1.

TABELA 1 -Coeficientes de Validade de Conteúdo (CVC) e Coeficiente de Concordância de Kappa: Clareza e Pertinência.

E: Experts IC: Intervalo de Confiança.

\begin{tabular}{lcccccc}
\hline \multirow{2}{*}{$\begin{array}{c}\text { Comparações } \\
\text { Experts }\end{array}$} & \multicolumn{3}{c}{ Clareza } & \multicolumn{3}{c}{ Pertinência } \\
\cline { 2 - 7 } & CVC & Kappa (IC 95\%) & $p$ & CVC & Kappa (IC 95\%) & $p$ \\
\hline E-1 $\times$ E-2 $\times$ E-3 & $97.91 \%$ & - & - & $98.25 \%$ & - & - \\
E-1 $\times$ E-2 & $98.23 \%$ & $0.85(0.80-0.96)$ & 0.001 & $98.91 \%$ & $0.89(0.80-0.95)$ & 0.001 \\
E-1 $\times$ E-3 & $98.80 \%$ & $0.87(0.82-0.91)$ & 0.001 & $99.20 \%$ & $0.90(0.82-0.98)$ & 0.001 \\
E-2 $\times$ E-3 & $99.20 \%$ & $0.93(0.84-0.99)$ & 0.001 & $100.00 \%$ & $0.94(0.85-1.00)$ & 0.001 \\
\hline
\end{tabular}

Validade de Face ou Aparente pelo Público-Alvo Adulto e Infantil

A validade aparente ou de face pelo públicoalvo adulto foi realizada com a participação de 26 profissionais utilizando-se a escala Likert. O percentual de concordância no total da escala foi de $97,5 \%$; nas sub-escalas os valores atingiram índices ainda mais elevados (competência cognitiva = 96\%; competência física $=98 \%$; aceitação dos pares $=97 \%$; aceitação materna $=99 \%)$. Em cada questão a concordância variou entre $95 \%$ e $100 \%$; a maioria dos profissionais pontuou escores máximos.

A validade aparente ou de face pelo públicoalvo infantil foi realizada com a participação de 20 meninos e meninas com idades entre 4 e 7 anos. Nenhuma das questóes sofreu modificaçóes uma vez que todas as crianças demonstraram entender as questóes e as responderam de forma apropriada; não questionando, em nenhum momento, o avaliador. As crianças maiores (6 e 7 anos), claramente expressaram que entendiam as questôes e rapidamente as respondiam. Uma criança de 4 anos em 3 momentos, solicitou a repetição da questão; após a repetição das questôes pelo pesquisador a criança respondeu de forma rápida e segura; demonstrando compreender a questáo.

\section{Medias, Desvio Padrão e Consistência Interna}

A TABELA 2 apresenta o número de crianças em cada grupo bem como as médias e desvios padrão para a PSPCSA e sub-escalas do presente estudo e de estudos prévios, para as comparações cross-culturais. No presente estudo não houve a observação de resultados extremos, o que possibilitou o uso dos procedimentos estatísticos adotados.

TABELA 2 -Média e Desvio Padrão: Subescalas e PSPCSA por Grupos (etapa de escolarização).

\begin{tabular}{|c|c|c|c|c|c|c|c|c|c|c|c|}
\hline \multirow{3}{*}{\multicolumn{2}{|c|}{$\begin{array}{c}\begin{array}{c}\text { Dimensões } \\
\text { \& Subescalas }\end{array} \\
\text { Grupos }\end{array}$}} & \multicolumn{10}{|c|}{ Cross-Cultural na PSPCSA \& Subescalas: M \& DP } \\
\hline & & \multicolumn{4}{|c|}{ Brasil $^{a}$} & \multicolumn{4}{|c|}{$\mathrm{USA}^{\mathrm{b}}$} & \multirow{2}{*}{$\frac{\text { Libano }^{c}}{\mathrm{~K}}$} & \multirow{2}{*}{$\begin{array}{c}\begin{array}{c}\text { Portu- } \\
\text { gal }^{\mathrm{d}}\end{array} \\
\mathrm{K}\end{array}$} \\
\hline & & EIG1 & EIG2 & EF1A & EF2A & $\mathrm{P}$ & K & G1 & G2 & & \\
\hline & & 99 & 139 & 224 & 195 & 90 & 56 & 65 & 44 & 152 & 30 \\
\hline \multicolumn{12}{|c|}{ Competência } \\
\hline \multirow{2}{*}{$\begin{array}{l}\text { Cogni- } \\
\text { tiva }\end{array}$} & M & 3.21 & 2.86 & 3.04 & 3.03 & 3.41 & 3.66 & 3.41 & 5.53 & 3.52 & 2.80 \\
\hline & DP & 0.71 & 1.34 & 1.35 & 1.27 & 0.40 & 0.40 & 0.43 & 0.31 & 0.41 & 0.37 \\
\hline \multirow{2}{*}{ Física } & M & 3.34 & 2.93 & 3.00 & 3.00 & 3.21 & 3.43 & 3.43 & 3.47 & 3.45 & 2.42 \\
\hline & $\mathrm{DP}$ & 0.73 & 1.32 & 1.23 & 1.23 & 0.52 & 0.35 & 0.40 & 0.41 & 0.53 & 0.42 \\
\hline \multirow{2}{*}{$\begin{array}{l}\text { Combi- } \\
\text { nada }\end{array}$} & $\mathrm{M}$ & 3.20 & 2.82 & 3.00 & 3.00 & 3.32 & 3.51 & 3.42 & 3.44 & 3.41 & - \\
\hline & DP & 0.71 & 1.32 & 1.21 & 1.23 & 0.42 & 0.33 & 0.44 & 0.34 & 0.43 & \\
\hline
\end{tabular}

continua 
continuação

\begin{tabular}{|c|c|c|c|c|c|c|c|c|c|c|c|}
\hline \multirow{3}{*}{\multicolumn{2}{|c|}{$\begin{array}{c}\begin{array}{c}\text { Dimensões } \\
\text { \& Subescalas }\end{array} \\
\text { Grupos }\end{array}$}} & \multicolumn{10}{|c|}{ Cross-Cultural na PSPCSA \& Subescalas: M \& DP } \\
\hline & & \multicolumn{4}{|c|}{ Brasil $^{\mathrm{a}}$} & \multicolumn{4}{|c|}{$\mathrm{USA}^{\mathrm{b}}$} & \multirow{2}{*}{$\frac{\text { Libano }^{c}}{K}$} & \multirow{2}{*}{$\begin{array}{c}\begin{array}{r}\text { Portu- } \\
\text { gal }^{\text {d }}\end{array} \\
\mathrm{K}\end{array}$} \\
\hline & & EIG1 & EIG2 & EF1A & EF2A & $\mathrm{P}$ & K & G1 & G2 & & \\
\hline \multicolumn{12}{|c|}{ Aceitação } \\
\hline \multirow{2}{*}{ Pares } & $\mathrm{M}$ & 3.11 & 2.62 & 2.82 & 2.85 & 3.00 & 2.96 & 3.12 & 3.11 & 2.90 & 3.21 \\
\hline & $\mathrm{DP}$ & 0.88 & 1.34 & 1.23 & 1.11 & 0.60 & 0.62 & 0.54 & 0.54 & 0.52 & 0.31 \\
\hline \multirow{2}{*}{ Física } & M & 3.04 & 2.43 & 2.42 & 2.12 & 3.12 & 2.91 & 2.87 & 2.87 & 3.22 & 2.72 \\
\hline & DP & 0.86 & 1.41 & 1.42 & 1.53 & 0.01 & 0.64 & 0.65 & 0.63 & 0.56 & 0.35 \\
\hline \multirow{2}{*}{$\begin{array}{l}\text { Combi- } \\
\text { nada }\end{array}$} & $\mathrm{M}$ & 3.00 & 2.52 & 2.60 & 2.43 & - & - & - & - & - & - \\
\hline & DP & 0.80 & 1.30 & 1.31 & 1.30 & & & & & & \\
\hline \multirow{2}{*}{$\begin{array}{l}\text { PSPC- } \\
\text { SA }\end{array}$} & $\mathrm{M}$ & 3.23 & 2.72 & 2.81 & 2.72 & - & - & - & - & - & - \\
\hline & DP & 0.71 & 1.30 & 1.20 & 1.10 & & & & & & \\
\hline
\end{tabular}

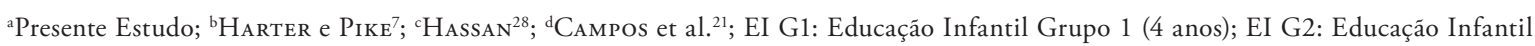
Grupo 2 (5 anos); EF 1A: Ensino Fundamental $1^{\circ}$ Ano; EF 2A: Ensino Fundamental 2Ano; P: Preschool; K: Kindergarten; 1G e 2G: First e Second Grade; - dado não informado.

A TABELA 3 apresenta os valores de alpha de Cronbach para consistência interna.

Os resultados evidenciam forte consistência da PSPCSA, bem como das 4 sub-escalas independentemente (valores de $\alpha>0,90$ ).

\section{Avaliação da Fidedignidade Teste-Reteste}

As correlações de Pearson, nos escores do teste-reteste para as sub-escalas e para PSPCSA total, foram significativas $(p=0,014)$, positivas, $\mathrm{e}$, variaram de moderadas à fortes (valores de $\mathrm{r}$ $>0,60)$.

Os resultados das correlações intra-classe foram ainda mais elevados. TABELA 4 apresenta os dados do presente estudo e os reportados em estudos prévios.

\section{Análise Fatorial Confirmatória}

No presente estudo foram examinados o modelo de quatro fatores (competência cognitiva, competência física, aceitação de pares e aceitação materna), teoricamente proposto por HARTER e PIKE 5 , e, o modelo de dois fatores (competência e aceitação) testado por HARTER e PIKE? Para os dois modelos as cargas fatoriais foram apropriadas. TABELA 5 apresenta esses resultados juntamente com os resultados de estudos prévios. A PSPCSA demonstrou índices apropriados de adequabilidade para os modelos de dois fatores (educação infantil: $\mathrm{NFI}=0,87 ; \mathrm{CFI}=$ $0,91 \mathrm{eTLI}=0,90 ; 1^{\circ} \mathrm{e} 2^{\circ}$ anos do ensino fundamental I: $\mathrm{NFI}=0,81 ; \mathrm{CFI}=0,83 \mathrm{eTLI}=0,81$ ) e de quatro fatores (educação infantil: $\mathrm{NFI}=0,93 ; \mathrm{CFI}=0,95 \mathrm{e}$ TLI $=0,95$; $1^{\circ}$ e $2^{\circ}$ anos do ensino fundamental I: NFI $=0,92 ; \mathrm{CFI}=$ $0,95$ e TLI $=0,95)$.

TABELA 3-Média e Desvio Padrão: Subescalas e PSPCSA por Grupos (etapa de escolarização).

\begin{tabular}{|c|c|c|c|c|c|c|c|c|c|c|c|}
\hline \multirow{2}{*}{$\begin{array}{l}\text { Dimen- } \\
\text { sões } \\
\& \\
\text { Subes- } \\
\text { calas }\end{array}$} & \multicolumn{11}{|c|}{ Cross Cultural $(\alpha)$ Consistência Interna } \\
\hline & \multicolumn{2}{|c|}{ Brasil $^{a}$} & \multicolumn{2}{|c|}{$\mathrm{USA}^{\mathrm{b}}$} & \multicolumn{2}{|c|}{$\mathrm{USA}^{\mathrm{c}}$} & \multicolumn{2}{|c|}{$\mathrm{USA}^{\mathrm{d}}$} & \multirow{2}{*}{$\begin{array}{c}\text { Libano }^{\mathrm{e}} \\
\mathrm{K}\end{array}$} & \multicolumn{2}{|c|}{ Portugual $^{\mathrm{f}}$} \\
\hline Grupos & $\begin{array}{c}\text { EIG1 + } \\
\text { G2 }\end{array}$ & $\begin{array}{c}\mathrm{EF} 1+ \\
2 \mathrm{~A}\end{array}$ & $\mathrm{~K}+\mathrm{P}$ & $\mathrm{G} 1+2$ & $\mathrm{~K}^{*}$ & $\mathrm{~K}^{* *}$ & G1 & G2 & & $\mathrm{KT}$ & K SD \\
\hline $\mathrm{N}$ & 238 & 419 & 146 & 109 & 133 & 251 & 199 & 109 & 152 & 30 & 47 \\
\hline \multicolumn{12}{|c|}{ Competência } \\
\hline $\begin{array}{l}\text { Cogni- } \\
\text { tiva }\end{array}$ & 0.93 & 0.94 & 0.67 & 0.76 & 0.50 & 0.43 & 0.73 & 0.71 & 0.52 & 0.33 & .85 \\
\hline Física & 0.93 & 0.93 & 0.62 & 0.53 & 0.57 & 0.52 & 0.48 & 0.58 & 0.59 & 0.59 & .88 \\
\hline
\end{tabular}

continua 
continuação

\begin{tabular}{|c|c|c|c|c|c|c|c|c|c|c|c|}
\hline \multirow{2}{*}{$\begin{array}{c}\text { Dimen- } \\
\text { sões } \\
\& \\
\text { Subes- } \\
\text { calas } \\
\end{array}$} & \multicolumn{11}{|c|}{ Cross Cultural $(\alpha)$ Consistência Interna } \\
\hline & \multicolumn{2}{|c|}{ Brasil $^{a}$} & \multicolumn{2}{|c|}{$\mathrm{USA}^{\mathrm{b}}$} & \multicolumn{2}{|c|}{$\mathrm{USA}^{\mathrm{c}}$} & \multicolumn{2}{|c|}{ USA $^{\mathrm{d}}$} & \multirow{2}{*}{$\begin{array}{c}\text { Libano }^{\mathrm{e}} \\
\mathrm{K}\end{array}$} & \multicolumn{2}{|c|}{ Portugual $^{\mathrm{f}}$} \\
\hline Grupos & $\begin{array}{c}\text { EIG1 + } \\
\text { G2 }\end{array}$ & $\begin{array}{c}\text { EF1 + } \\
2 \mathrm{~A}\end{array}$ & $\mathrm{~K}+\mathrm{P}$ & $\mathrm{G} 1+2$ & $\mathrm{~K}^{*}$ & $\mathrm{~K}^{* *}$ & G1 & G2 & & $\mathrm{KT}$ & K SD \\
\hline $\mathrm{N}$ & 238 & 419 & 146 & 109 & 133 & 251 & 199 & 109 & 152 & 30 & 47 \\
\hline \multicolumn{12}{|c|}{ Competência } \\
\hline $\begin{array}{l}\text { Combi- } \\
\text { nada }\end{array}$ & 0.96 & 0.93 & 0.76 & 0.77 & 0.71 & 0.63 & - & - & 0.70 & - & - \\
\hline \multicolumn{12}{|c|}{ Aceitação } \\
\hline Pares & 0.92 & 0.92 & 0.74 & 0.79 & 0.74 & 0.75 & 0.81 & 0.87 & 0.72 & 0.39 & .82 \\
\hline $\begin{array}{l}\text { Mater- } \\
\text { na }\end{array}$ & 0.94 & 0.96 & 0.83 & 0.74 & 0.80 & 0.75 & - & - & 0.31 & 0.35 & .44 \\
\hline $\begin{array}{l}\text { Combi- } \\
\text { nada }\end{array}$ & 0.95 & 0.95 & 0.87 & 0.86 & 0.86 & 0.84 & - & - & 0.68 & - & - \\
\hline $\begin{array}{l}\text { PSPC- } \\
\text { SA }\end{array}$ & 0.97 & 0.97 & 0.88 & 0.87 & 0.87 & 0.83 & 0.83 & 0.81 & - & - & - \\
\hline
\end{tabular}

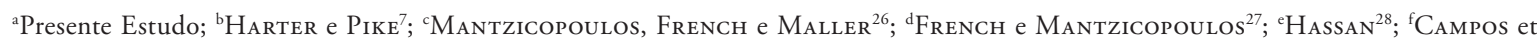
al. ${ }^{21}$; EIG1+G2: Educaçáo Infantil Grupo 1 (4 anos) + Grupo 2 (5 anos); EF1+2A: Ensino Fundamental $1^{\circ}+2^{\circ}$ Anos; P+K: Preschool + Kindergarten; $\mathrm{K}^{*}$ e $\mathrm{K}^{* *}$ : Kindergarten crianças em desvantagem econômica* e de classe media*; G1+2: Grades -First Grade + Second Grade; T: típicas. SD: síndrome de Down; - dado não informado.

TABELA 4-PSPCSA e Subescalas Fidedignidade Teste-Reteste:Validação Brasileira e Americana.

\begin{tabular}{|c|c|c|c|c|c|c|}
\hline \multirow{4}{*}{$\begin{array}{c}\text { Dimensões } \\
\text { \& Subescalas }\end{array}$} & \multicolumn{6}{|c|}{ Cross Cultural $(\alpha)$ Fidedignidade Teste-Reteste } \\
\hline & \multicolumn{4}{|c|}{ Brasil $^{a}$} & \multirow{3}{*}{$\begin{array}{c}\mathrm{USA}^{\mathrm{b}} \\
\mathrm{K}(117) \\
\alpha\end{array}$} & \multirow{3}{*}{$\begin{array}{c}\text { Portugual } \\
\text { K SD (12) } \\
\mathrm{r}\end{array}$} \\
\hline & \multicolumn{2}{|c|}{ EIG1+G2 (59) } & \multicolumn{2}{|c|}{$\mathrm{EF} 1+2 \mathrm{~A}(100)$} & & \\
\hline & $\mathrm{r}(p)$ & $\alpha$ & $\mathrm{r}(p)$ & $\alpha$ & & \\
\hline \multicolumn{7}{|l|}{ Competência } \\
\hline Cognitiva & $0.60(0.014)$ & 0.68 & $0.75(0.0001)$ & 0.85 & 0.59 & - \\
\hline Física & $0.67(0.001)$ & 0.76 & $0.74(0.0001)$ & 0.86 & 0.67 & - \\
\hline Combinada & $0.75(0.001)$ & 0.80 & $0.78(0.0001)$ & 0.90 & - & 0.70 \\
\hline \multicolumn{7}{|l|}{ Aceitação } \\
\hline Pares & $0.60(0.002)$ & 0.88 & $0.79(0.0001)$ & 0.89 & 0.77 & - \\
\hline Materna & $0.78(0.001)$ & 0.88 & $0.78(0.0001)$ & 0.90 & 0.74 & - \\
\hline Combinada & $0.82(0.001)$ & 0.90 & $0.80(0.0001)$ & 0.91 & - & 0.81 \\
\hline PSPCSA & $0.73(0.001)$ & 0.90 & $0.81(0.0001)$ & 0.89 & - & \\
\hline
\end{tabular}

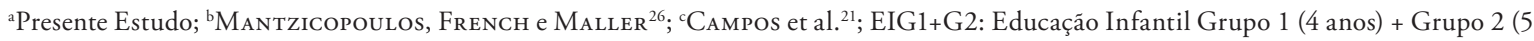
anos); EF1+2A: Ensino Fundamental $1^{\circ}+2^{\circ}$ Anos; K: Kindergarten; KSD: Kindergarten com Síndrome de Down; - não reportado. 
TABELA 5-PSPCSA Dimensões e Subescalas Cross-cultural Fatorial Carga: Brasil e Estados Unidos.

\begin{tabular}{|c|c|c|c|c|c|c|c|c|c|c|}
\hline \multirow{2}{*}{\multicolumn{2}{|c|}{$\begin{array}{l}\text { Questões/ Subes- } \\
\text { calas }\end{array}$}} & \multicolumn{2}{|c|}{$\begin{array}{c}\text { Brazil }^{a} \\
4 \text { fatores }\end{array}$} & \multicolumn{2}{|c|}{$\begin{array}{c}\text { USA }^{b} \\
3 \text { fatores }\end{array}$} & \multirow{2}{*}{$\begin{array}{c}\text { Ques- } \\
\text { tões/ } \\
\text { Dimen- } \\
\text { são }\end{array}$} & \multicolumn{2}{|c|}{$\begin{array}{c}\text { Brazil }^{\mathrm{a}} \\
2 \text { fatores }\end{array}$} & \multicolumn{2}{|c|}{$\begin{array}{c}\text { USA }^{c} \\
2 \text { fatores }\end{array}$} \\
\hline & & $\begin{array}{c}\text { EIG1 + } \\
\text { G2 }\end{array}$ & $\begin{array}{c}\text { EF1 + } \\
2 A\end{array}$ & G1 & G2 & & \multirow{2}{*}{$\begin{array}{c}\text { EIG1 + } \\
\text { G2 } \\
0.85\end{array}$} & \multirow{2}{*}{$\frac{\mathrm{EF} 1+2}{0.84}$} & \multirow{2}{*}{$\frac{\mathbf{P}+\mathbf{K}}{0.39}$} & \multirow{2}{*}{$\frac{\mathbf{G 1}+2}{0.51}$} \\
\hline $\mathrm{N}^{\circ} 1$ & $\operatorname{Cog} n$ & 0.85 & 0.83 & 0.58 & 0.50 & Comp & & & & \\
\hline No 5 & $\operatorname{Cog} n$ & 0.74 & 0.87 & 0.75 & 0.66 & Comp & 0.74 & 0.86 & 0.37 & 0.63 \\
\hline $\mathrm{N}^{\circ} 9$ & $\operatorname{Cog} n$ & 0.85 & 0.86 & 0.62 & 0.68 & Comp & 0.85 & 0.85 & 0.57 & 0.50 \\
\hline $\mathrm{N}^{0} 13$ & $\operatorname{Cog} n$ & 0.89 & 0.91 & 0.80 & 0.76 & Comp & 0.90 & 0.89 & 0.43 & 0.65 \\
\hline No 17 & $\operatorname{Cog} n$ & 0.83 & 0.88 & 0.48 & 0.67 & Comp & 0.83 & 0.87 & 0.48 & 0.51 \\
\hline $\mathrm{N}^{\circ} 21$ & $\operatorname{Cog} n$ & 0.85 & 0.88 & 0.66 & 0.64 & Comp & 0.85 & 0.87 & 0.58 & 0.40 \\
\hline $\mathrm{N}^{\circ} 3$ & Física & 0.90 & 0.86 & 0.34 & 0.22 & Comp & 0.90 & 0.85 & 0.19 & 0.22 \\
\hline $\mathrm{N}^{\circ} 7$ & Física & 0.90 & 0.86 & 0.57 & 0.64 & Comp & 0.90 & 0.84 & 0.33 & 0.48 \\
\hline $\mathrm{N}^{0} 11$ & Física & 0.64 & 0.78 & 0.67 & 0.42 & Comp & 0.64 & 0.76 & 0.42 & 0.43 \\
\hline $\mathrm{N}^{\circ} 15$ & Física & 0.90 & 0.90 & 0.60 & 0.26 & Comp & 0.89 & 0.89 & 0.34 & 0.33 \\
\hline No 19 & Física & 0.93 & 0.90 & 0.71 & 0.54 & Comp & 0.92 & 0.89 & 0.23 & 0.50 \\
\hline $\mathrm{N}^{\circ} 23$ & Física & 0.84 & 0.81 & 0.53 & 0.53 & Comp & 0.84 & 0.81 & 0.22 & 0.40 \\
\hline $\mathrm{N}^{\circ} 2$ & Pares & 0.88 & 0.86 & 0.73 & 0.63 & Aceit & 0.82 & 0.65 & 0.36 & 0.67 \\
\hline $\mathrm{N}^{\circ} 6$ & Pares & 0.70 & 0.70 & 0.68 & 0.63 & Aceit & 0.71 & 0.65 & 0.47 & 0.27 \\
\hline $\mathrm{N}^{\circ} 10$ & Pares & 0.88 & 0.89 & 0.82 & 0.78 & Aceit & 0.84 & 0.68 & 0.23 & 0.60 \\
\hline $\mathrm{N}^{\circ} 14$ & Pares & 0.89 & 0.87 & 0.89 & 0.82 & Aceit & .0 .85 & 0.63 & 0.36 & 0.67 \\
\hline $\mathrm{N}^{\circ} 18$ & Pares & 0.78 & 0.87 & 0.85 & 0.72 & Aceit & 0.77 & 0.70 & 0.44 & 0.72 \\
\hline $\mathrm{N}^{\circ} 22$ & Pares & 0.76 & 0.76 & 0.84 & 0.77 & Aceit & 0.76 & 0.72 & 0.61 & 0.67 \\
\hline $\mathrm{N}^{\circ} 4$ & Materna & 0.84 & 0.87 & - & - & Aceit & 0.80 & 0.85 & 0.52 & 0.44 \\
\hline $\mathrm{N}^{\circ} 8$ & Materna & 0.92 & 0.94 & - & - & Aceit & 0.87 & 0.92 & 0.52 & 0.58 \\
\hline $\mathrm{N}^{\circ} 12$ & Materna & 0.86 & 0.92 & - & - & Aceit & 0.80 & 0.90 & 0.53 & 0.63 \\
\hline $\mathrm{N}^{\circ} 16$ & Materna & 0.79 & 0.89 & - & - & Aceit & 0.76 & 0.88 & 0.61 & 0.61 \\
\hline $\mathrm{N}^{\circ} 20$ & Materna & 0.84 & 0.86 & - & - & Aceit & 0.80 & 0.85 & 0.70 & 0.51 \\
\hline $\mathrm{N}^{\circ} 24$ & Materna & 0.84 & 0.92 & - & - & Aceit & 0.81 & 0.90 & 0.62 & 0.50 \\
\hline
\end{tabular}

Cogn: Cogniçãa; Comp: Competência; Aceit: Aceitação; ${ }^{a}$ Presente Estudo: $n=647$; ${ }^{b}$ French e Mantzicopoulos ${ }^{27}: \mathrm{n}=308$; ${ }^{\mathrm{H}} \mathrm{HarTER}$ e PIKE 7 n = 245; C: Competência; A: Aceitação; EIG1+G2: Educação Infantil Grupo 1 (4 anos) + Grupo 2 (5 anos); EF1+2A: Ensino Fundamental 1 ${ }^{\circ}+2^{\circ}$ Anos; P+K: Preschool + Kindergarten; G1+2: Grades -First Grade + Second Grade; -- a subescala aceitação materna não foi aplicada no estudo.

\section{Discussão}

\section{Dupla Tradução Reversa e Independente}

A tradução do PSPCSA foi realizada seguindo critérios rigorosos de tradução dupla, reversa $\mathrm{e}$ independente recomendados na literatura psicométrica referente à adaptação cross-cultural de instrumentos. $\mathrm{O}$ procedimento adotado no presente estudo, com quatro tradutores, permitiu minimizar distorções de tradução observadas quanto a tradução de um instrumento, para outra cultura, é realizada por um único tradutor ${ }^{37}$.

\section{Avaliação dos Experts e Público-Alvo Adulto e Infantil}

Os experts consideraram o conteúdo da PSPCSA apropriado para avaliar as percepções de competência cognitiva e física da criança, bem como das percepçõoes de aceitação materna e de pares. Os resultados do CVC (superiores a 0,97), para a clareza e a pertinência, demonstraram a coerência elevada entre os experts, acima do ponto de corte mais rigoroso de $0,80^{36,40,41}$. Respostas 
similares e elevada concordância entre os experts foi confirmada pelo teste de Kappa ${ }^{36,40}$. Os resultados evidenciam que a tradução e representação das questões da escala são adequadas em relação à base conceitual e teórica adotada ${ }^{33,36,40}$.

A validade aparente ou de face foi realizada com o público-alvo adulto os quais também consideraram o conteúdo da PSPCSA claro e pertinente para avaliar a competência percebida e aceitação social de crianças. A clareza das questôes foi examinada também pelo público-alvo infantil. Nesta etapa, com o público-alvo infantil, a preocupação principal dos pesquisadores foi verificar se o uso de linguagem nas questôes da escala era acessível e claro para as crianças, e se as mesmas entendiam as questôes ${ }^{37,40}$. A adoção deste procedimento evidencia a rigorosidade e adequação do processo de validação adotado no presente estudo; e, reflete também a clareza e simplicidade das questóes da PSPCSA, uma vez que não houve dúvidas por parte de nenhuma criança.

\section{Confiabilidade das Medias e Consistência Interna}

As médias observadas no presente estudo são confiáveis, sem adesão aos extremos, e sem ocorrência do fenômeno da aquiescência, quer positiva ou negativa, portanto as mesmas são representativas das questôes contidas na escala ${ }^{33,41}$, resultado semelhante foi previamente reportado em estudos prévios em outros países ${ }^{7,21,28}$. As médias foram mais elevadas para crianças de 4 anos da educação infantil, crianças nesta faixa etária tendem a expressar competências superestimadas ${ }^{7,25,28}$, fenômeno observado no presente estudo. Nas comparações cross-culturais, as crianças brasileiras apresentaram médias mais baixas que crianças Americanas $^{5,7}$ e Libanesas ${ }^{28}$; e, similares as médias reportadas para crianças Portuguesas ${ }^{21}$.

Os resultados da análise do alpha de Cronbach (acima de 0,90) evidenciam um perfil de homogeneidade e adequada consistência interna da PSPCSA para crianças brasileiras, uma vez que valores acima de 0,60 são aceitos como apropriados ${ }^{33,44}$. Os resultados do alpha de Cronbach, do presente estudo, foram mais elevados do que os resultados reportados para crianças americanas no estudo de validação original ${ }^{7}$ e para crianças Americanas em estudos mais recentes ${ }^{26,27}$; bem como, aos reportados para crianças Libanesas ${ }^{28}$ e Portuguesas com e sem síndrome de Down ${ }^{21}$.

\section{Fidedignidade Teste-Reteste}

A fidedignidade, em termos de estabilidade temporal, foi confirmada para as sub-escalas e para a PSPCSA, todos os valores foram acima de 0,60 nas correlaçóes teste-reteste, resultados considerados desejáveis e apropriados ${ }^{33,36,40,41,43}$. No domínio de aceitação (pares e materna) os índices foram mais elevados que no domínio das competências (cognitiva e física). Ainda mais, para a PSPCSA de crianças nos anos iniciais obtevese resultados mais elevados quando comparados aos escores da PSPCSA de crianças na educação infantil. Em amostras grandes, como a do presente estudo, a não observação de instabilidade temporal evidencia a forte fidedignidade da escala nas crianças investigadas $s^{34,40,43}$, fator essencial para o uso da mesma no Brasil. Ainda mais, os resultados reportados no presente estudo são mais elevados do que os resultados reportados com crianças Americanas ${ }^{25-27}$, e, similares aos resultados reportados para crianças Portuguesas ${ }^{21}$.

\section{Análise Fatorial Confirmatória}

A validade de construto foi também mensurada utilizando-se de análise fatorial confirmatória ${ }^{43,45}$ para o modelo de dois fatores (dimensão: competência ou aceitação) e de quatro fatores (subescalas: competência cognitiva e física, aceitação de pares e materna). Cada questão se relacionou de forma adequada com a respectiva dimensão ou sub-escala. As cargas fatoriais foram adequadas e os índices de confiabilidade elevados nos dois modelos, sendo mais fortes no modelo de quatro fatores. Não foram observadas relações negativas entre as questôes e as dimensões ou sub-escalas, resultados que propiciam suporte aos modelos analisados ${ }^{34}$.

Todos os índices de adequabilidade ao modelo (NFI, CFI e TLI) foram superiores ao valor rigoroso adotado no presente estudo $(0,80)$, e, portanto, fortemente apropriados ${ }^{45,46}$. Os resultados do presente estudo quanto às duas dimensões são considerados (modelo de dois fatores) são mais fortes. Estes resultados se alinha com os apresentados por HARTER e PIKE ${ }^{7}$, na observância das duas dimensões (competência e aceitação) para a escala. Entretanto, se diferenciam dos reportados para crianças Libanesas ${ }^{28}$ no qual para o modelo de dois fatores agruparam-se competência cognitiva e física e aceitação social em um fator, e o segundo 
fator composto somente pela aceitação materna.

Quanto ao modelo de 4 fatores, os resultados do presente estudo são adequados quanto a carga fatorial e índices de adequabilidade ${ }^{45,46}$. Ainda mais, estes resultados, quanto à carga fatorial, são mais elevados que os reportados para crianças Americanas com modelo de 3 fatores no estudo de FrENCH e Mantzicopoulos ${ }^{27}$. Os dois estudos, o presente e o americano, demonstram índices de adequabilidade de modelo e alinhamento das questões nas respectivas sub-escalas de forma semelhante; embora, caiba destacar, que o estudo americano não investigou as propriedade psicométricas da sub-escala de aceitação materna ${ }^{27}$, fator que limita em parte as comparaçõos. Ainda mais, índices de adequabilidade não foram propiciados por HARTER e PIKE ${ }^{7}$ para o modelo de 4 fatores o que impede a comparação dos mesmos. Entretanto, as autoras sugerem que a carga fatorial no modelo de dois fatores garantem também, sob o ponto de vista teórico, o uso das escalas independentes (modelo de 4 fatores), resultado que no presente estudo foi evidenciado com a análise fatorial confirmatória. Portanto, o presente estudo propicia evidencias para o modelo de 4 fatores, o qual até o momento era suportado apenas por teoria.

Concluindo, os resultados nos permitem reportar que a concordância entre experts e profissionais, e a consistência interna foram elevadas e positivas, propiciando evidências favoráveis a validade de critério e conteúdo da PSPCSA ${ }^{34}$. Além disso, os resultados da estabilidade temporal evidenciou a fidedignidade da escala ${ }^{33,36,40,41}$. Também foram encontradas evidências para o constructo de duas dimensões, bem como para o modelo de quatro sub-escalas independentes (um avanço nas evidencias reportadas até então), considerando a adequada carga fatorial das questōes nas dimensões e nas sub-escalas ${ }^{45,46}$. Portanto, os resultados permitem assumir a validade do conteúdo, critério e constructo, bem como a fidedignidade da PSPCSA para crianças brasileiras na educação infantil ( 4 e 5 anos) e do $1^{\circ}$ e $2^{\circ}$ anos do ensino fundamental I (6 e 7 anos); podendo a mesma ser usada na avaliação tanto das dimensões de competência e aceitação quanto nas quatro sub-escalas.

Diversos procedimentos foram adotados no presente estudo para verificar a adequabilidade do uso das duas versões da PSPCSA (para crianças no educação infantil e dos primeiros dois anos do ensino fundamental I). A tradução mostrou-se adequada e resultados apropriados foram encontrados para consistência interna e fidedignidade. Ainda mais, no presente estudo confirmamos o modelo teórico ( 4 fatores) e empírico (2 fatores) apresentados para a versão original da PSPCSA. Destacamos que até o presente não encontramos nenhum estudo que houvesse testado e propiciado suporte ao modelo de 4 fatores teoricamente proposto no estudo original da PSPCSA, contribuição original do presente estuo. As versões da PSPCSA (educação infantil e nos primeiros dois anos do ensino fundamental I) são adequadas para uso com crianças brasileiras entre 4 e 7 anos de idades.

\title{
Agradecimentos
}

Fontes de financiamento: CNPq e CAPES.

\begin{abstract}
Validity and Reliability of the Pictorial Scale of Perceived Competence and Social Acceptance for Brazilian

The Pictorial Scale of Perceived Competence and Social Acceptance (PSPCSA) is a scale used to identify self-perceptions of competence (i.e., cognitive and physical) and social acceptance (i.e., peers and maternal) of children in kindergarten, first and second grades of elementary school. The objective of this study was to translate and investigate the content, criteria and construct validity, and reliability of the PSPCSA for Brazilian children. The cross-cultural translation was used, and the process of validation was conducted involving 33 professionals and 667 children (4 to 7 years-old). On two occasions, 159 children answered the PSPCSA. The results revealed that the scale has clear and pertinent questions, high internal consistence
\end{abstract}


(values $>0.96$ ) and test-retest reliability ( $r$ values $>0.60$ ). The PSPCSA showed adjusted adhesion of the questions with the correspondent dimensions and subscales, and satisfactory indices of the confirmatory factorial validity (Non-normed Fit Index, Comparative Fit Index and Tucker e Lewis's Index of Fit with values $>$ 0.81). The Brazilian versions of PSPCSA are valid and reliable to be used with Brazilian children.

KEYwORDS: PSPCSA; Pictorial Scale of Self-Perceptions; Validation Study.

\section{Referências}

1. Harter S. The construction of the self: Developmental and sociocultural foundations. New York: Guilford Press; 2012.

2. Harter S. Emerging self processes during childhood and adolescence. In: Leary M, Tangney J, organizadores. Handbook of self and identity. New York: Guilford Press; 2012.

3. Harter S. The construction of the self: A developmental perspective. New York: The Guilford Press; 1999.

4. Jumbunathan S, Norris JA. Perception of self-competence in relation to language competence among preschoolers. Child Stud J. 2000;30:91-101.

5. Harter S, Pike R. The pictorial scale of perceived competence and social acceptance for young children. Denver: University of Denver Press; 1981.

6. Harter S. New directions in self-development: Resurrecting the I-self. In: McInerney D, organizador. Theory driving research: New wave perspectives on self-processes and human development. New York: Information Age Publishing; 2012.

7. Harter S, Pike R. The pictorial scale of perceived competence and social acceptance for young children. Child Dev. 1984;55:1969-1982. doi:10.2307/1129772.

8. DiBiase R, Miller P. Self-perceived peer acceptance in preschoolers of differing economic and cultural backgrounds. J Genet Psychol. 2015;176:139-155. doi:10.1080/00221325.2015.1022504.

9. Coplan RJ, Findlay LC, Nelson LJ. Characteristics of preschoolers with lower perceived competence. J Abnorm Child Psychol. 2004;32:399-408.

10. Donnellan MB, Trzesniewski KH, Robins RW, Moffitt TE, Caspi A. Low self-esteem is related to aggression, antisocial behavior, and delinquency. Psychol Sci. 2005;16:328-335. doi: 10.1111/j.0956-7976.2005.01535.x.

11. Ekornås B, Lundervold AJ, Tjus T, Heimann M. Anxiety disorders in 8-11-year-old children: Motor skill performance and self-perception of competence. Scand J of Psychol. 2010;51:271-277. doi: 10.1111/j.1467-9450.2009.00763.x.

12. Hughes JN, Zhang D. Effects of the structure of classmates' perceptions of peers' academic abilities on children's perceived cognitive competence, peer acceptance, and engagement. Contemp Psychol. 2005;32:440-419. doi: 10.1016/j. cedpsych.2005.12.003.

13. Taylor M, Carlson SM, Maring BL, Gerow L, Charley CM. The Characteristics and correlates of fantasy in school-age children: Imaginary companions, impersonation, and social understanding. Dev Psychol. 2004;40:1173-1187. doi: org/10.1037/0012-1649.40.6.1173.

14. Altermatt ER, Pomerantz EM. The development of competence-related and motivational beliefs: an investigation of similarity and influence among friends. J Educ Psychol. 2003;95:111-123. doi: org/10.1037/0022-0663.95.1.111.

15. Barber L, Grubbs B, Cottrell S. Self-perception in children with attention deficit/ hyperactivity disorder. J Pediatr Nurs. 2005;20:235-245. doi:10.1016/j.pedn.2005.02.012.

16. Chen X, Zappulla C, Lo Coco A, Schneider B, Kaspar V, Oliveira AM, et al. Self-perceptions of competence in Brazilian, Canadian, Chinese and Italian children: relations with social and school adjustment. Int J Behav Dev. 2004;28:129138. doi: 10.1080/01650250344000334.

17. Heath NL, Glen T. Positive illusory bias and the self-protective hypothesis in children with learning disabilities. J Clin Child Adolesc Psychol. 2005;34:272-281.

18. Valentini NC, Rudisill ME. An inclusive mastery climate intervention on the motor skill development of children. Adapt Phys Activ Q. 2004;21:330-347. 
19. Valentini NC, Rudisill ME. Motivational climate, motor-skill development, and perceived competence: Two studies of developmentally delayed kindergarten children. J Teach Phys Educ. 2004;23:216-234.

20. Hassandra M, Goudas M, Chroni S. Examining factors associated with intrinsic motivation in physical education: A qualitative approach. Psychol Sport Exerc. 2003;4:211-223. doi:10.1016/S1469-0292(02)00006-7.

21. Campos JC, Ferreira JP, Gaspar PM, Gorla JI. Perceived competence and social acceptance assessment in Portuguese young students with and without Down syndrome: a preliminary analysis. Eur J Adap Phys Act. 2008;1:51-61.

22. Cugmas Z. The external validity of the scale of self-perceptions for pre-school children (LSPO). In: Columbus AM, organizador. Leading Edge: Research in Cognitive Psychology. New York: Nova Science Publishers Inc; 2006.

23. Byrne BM. Measuring self-concept across the life span: Issues and instrumentation. Measurement and instrumentation in psychology. Washington, DC: American Psychological Association; 1996.

24. Jambunathan S, Burtis D. Comparison of perception of self-competence among five ethnic groups of preschoolers in the US. Early Child Dev Care. 2003;173:651-660. doi: 10.1080/0300443032000088249.

25. Mantzicopoulos P. I am really good at puzzles, but I don't get asked to play with others: Age, gender, and ethnic differences in head start children's self-perceptions of competence. J Genet Psychol. 2004;165:51-65. doi: 10.3200/ GNTP.165.1.51-66.

26. Mantzicopoulos P, French BF, Mailer SJ. Factor structure of the pictorial scale of perceived competence and social acceptance with two pre-elementary samples. Child Dev. 2004;75(4):1214-28.

27. French BF, Mantzicopoulos P. An examination of the first/second-grade form of the pictorial scale of perceived competence and social acceptance: Factor structure and stability by grade and gender across groups of economically disadvantaged children. J Sch Psychol. 2007;45:311-331. doi:10.1016/j.jsp.2006.07.004.

28. Hassan KE. Validation of the Harter pictorial scale of perceived competence and social acceptance with Lebanese children. Soc Behav Pers. 1999;27:339-354. doi: org/10.2224/sbp.1999.27.4.339.

29. Veenhof C. The pictorial scale of perceived competence and social acceptance for children with cerebral Palsy: A reliability and validation study. Amsterdam (AFI): Vrije Universiteit; 1994.

30. Valentini NC, Vilwock G, Vieira LF, Vieira JLL, Barbosa MLL. Validação brasileira da escala de autopercepção de Harter para crianças. Psicol: Reflex Crit. 2010;23:411-419.

31. Bandeira DR, Arteche AX, Reppold CT. Escala de Autopercepção de Harter para Adolescentes: Um Estudo de Validação. Psic: Teor e Pesq. 2008;24:341-345.

32. Valentini NC. Percepçóes de Competência e Desenvolvimento Motor de meninos e meninas: um estudo transversal. Movimento. 2002;8:51-62.

33. Cronbach LJ. Fundamentos da testagem psicológica. Porto Alegre: Artes Médicas; 1996.

34. Vallerand JR. Vers une mèthodologie de validation trans-culturelle de questionnaires psychologiques: implications pour la recherche en langue fraçaise. Canadian Psychology. 1989;30:662-680.

35. Dassa C. Analyze multidimensionnelle exploratoire et confirmative. Montreal: Univesité Montreal; 1999.

36. Hernandez-Nieto R. Contributions to statistical analysis. Mérida: Los Andes University Press; 2002.

37. Boomsma KA. The robustness of LISREL against small sample size in factor analysis models. In: Jöreskog KG, Wold $\mathrm{H}$, organizadores . Systems under indirect observation: Causality, structure, prediction. Amsterdam: North Holland; 1982. p. 149-173.

38. Harter S, Pike R. Procedural Manual to Accompany: The Pictorial Scale of Perceived Competence and Social acceptance for young children. Denver: University of Denver Press; 1983.

39. Neuendorf KA. The content analysis guidebook. London: SAGE Publication; 2002.

40. Bryman A, Cramer D. Quantitative data analysis with SPSS release perceived symptoms - Criterion validity of the Toronto Alexithymia LOS8 for Windows: A guide for social scientists. New York: Routledge; 1999.

41. Kline P, Saggino A. Item factor analysis of the Italian version of the Myers-Briggs Type Indicator Source. Pers Individ Dif. 1995;19:243-249.

42. Sim J, Wright C. Research in health care: concepts, designs and methods. United Kingdom: Nelson Thomes; 2000.

43. Berry JW, Poortinga YH, Segall MH, Dasen PR. Cross-cultural. Psychology: Research and applications. Cambridge: Cambridge University Press; 1992. 
Valentini NC, et al.

44. Breakweell GM, Hammond S, Fife-Schaw C, Smith JA. Research Methods in Psychology. London: SAGE Publication Ltd; 2006.

45. Taylor GJ, Bagby RM, Parker JDA. The 20-Item Toronto Alexithymia Scale IV. Realiability and factorial validity in different languages and cultures. J Psychol Res. 2003;55:277-283. doi: 10.1016/S0022-3999(02)00601-3.

46. Tucker S, Lewis C. A reliability coefficient for maximum likelihood factor analysis. Psychometrika. 1973;38:1-10. doi: 10.1007/BF02291170.

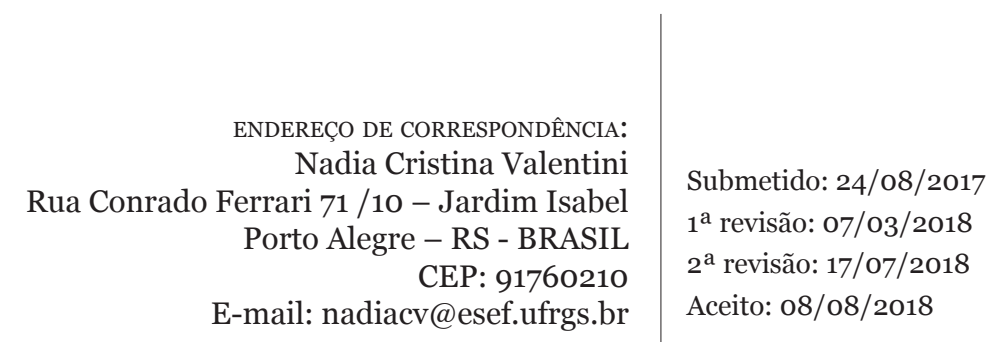

